\title{
On the Total Bandwidth for the Rational Harper's Equation
}

\author{
Bernard Helffer ${ }^{1}$, Phillippe Kerdelhué ${ }^{2}$ \\ ${ }^{1}$ DMI-Ecole Normale Supérieure, 45 rue d'Ulm, F-75230 Paris Cédex, France. URA CNRS 762 \\ 2 Université de Paris-Sud, Département de Mathématiques, F-91405 Orsay Cédex, France. URA \\ CNRS 760
}

Received: 2 August 1994/in revised form: 1 December 1994

\begin{abstract}
In the last years several contributions have been done around the total bandwidth of the spectrum for the Harper's operator. In particular an interesting conjecture has been proposed by Thouless which gives also strong convincing arguments for the proof in special cases. On the other hand, in the study of the Cantor structure of the spectrum, B. Helffer and J. Sjöstrand have justified an heuristic semiclassical approach proposed by M. Wilkinson. The aim of this article is to explain how one can use the first step of this approach to give a rigorous semi-classical proof of the Thouless formula in some of the simplest cases. We shall also indicate how one can hope with more effort to prove rigorously recent results of Last and Wilkinson on the same conjecture.
\end{abstract}

\section{Introduction}

The Harper's operator $H_{x, \lambda, \theta}$ is defined on $l^{2}(\mathbb{Z})$ as

$$
\left(u_{n}\right)_{n \in \mathbb{Z}} \rightarrow\left(H_{\alpha, i, \theta} u\right)_{n}=\frac{1}{2}\left(u_{n+1}+u_{n-1}\right)+\lambda \cos (2 \pi \alpha n+\theta) u_{n} .
$$

Here $\alpha$ and $\theta$ are real parameters satisfying:

$$
0 \leqq \alpha \leqq 1, \quad 0 \leqq \theta \leqq 2 \pi .
$$

The most interesting spectral properties of this operator appear when $\alpha$ is irrational. In this case a Cantor structure for the spectrum is expected. As was observed by D. Hofstadter who get a beautiful butterfly [16], we get a good intuition of the problem by a careful analysis of the spectrum in the rational case. In this case:

$$
\alpha=p / q
$$

and the spectrum $\sigma(\alpha, \lambda, \theta)$ of the Harper's operator depends effectively on $\theta$ and one more interesting spectrum is the closed set:

$$
\Sigma(\alpha, \lambda)=\operatorname{def} \bigcup_{0 \in[0,2 \pi]} \sigma(\alpha, \lambda, \theta)
$$


which appears to be the union of $q$ bands which are essentially disjoint outside the case when $q$ is even. In this case the two central bands are touching at 0 . This property was first observed by Hofstadter [16] and rigorous proofs of these properties were obtained for generic $\lambda$ in [6] and finally for any $\lambda$ in [22] (see also the nice proof by [10]). For an historical presentation of the subject we refer to the surveys of J. Bellissard [4,5]. A selected collection of zooms inside the butterfly is presented in [11].

Following heuristic semi-classical ideas of M. Wilkinson [34], B. Helffer and J. Sjöstrand have developed in $[12,13,14]$ and with P. Kerdelhué [15] a machinery which permits to prove in the case $\lambda=1$ and for special sequences of irrationals $\alpha$ whose properties are explicitly described in terms of their expansion in continuous fraction, the Cantor structure of the spectrum. What is sometimes overlooked is that the first step in the proof has a very general character and is adapted to large classes of $h$-pseudodifferential operators. By $h$-pseudodifferential operators we mean operators which can be defined on the Schwartz space $\mathscr{S}(\mathbb{R})$ by the formula:

$$
\left(p\left(x, h D_{x}\right) u\right)(x)=(2 \pi h)^{-1} \int_{\mathbb{R}} \int_{\mathbb{R}} \exp i((x-y) \xi / h) p((x+y) / 2, \xi, h) u(y) d y d \xi,
$$

where $p$ is a $C^{\infty}$-function on $\mathbb{R}^{2}$ which is in our context $2 \pi$-periodic in the $x$ and $\xi$ variable $\left((x, \xi) \in \mathbb{R}^{2}\right)$. In this formalism, the set which was introduced in (1.3) can be identified (for any $\alpha$ ) as the spectrum of the $h$-pseudodifferential operator:

$$
H_{\alpha, \lambda}=H_{\lambda}\left(x, h D_{x}\right)=\lambda \cos \left(h D_{x}\right)+\cos x,
$$

considered as a selfadjoint bounded operator on $L^{2}(\mathbb{R})$, with $h=2 \pi \alpha$, whose symbol is the function $(x, \xi) \rightarrow \lambda \cos \xi+\cos x$.

As a byproduct of a careful semi-classical analysis and of an infinite sequence of approximate renormalizations, the authors get the following theorem:

Theorem 1.1. There exists a constant $C \geqq 1$ such that if $\alpha$ is an irrational admitting a decomposition in continuous fraction:

$$
\alpha=\left[q_{1}, q_{2}, \ldots, q_{n}, \ldots\right]={ }^{\operatorname{def}} \frac{1}{q_{1}+\frac{1}{q_{2}+\frac{1}{q_{3}+\cdots}}}
$$

with

$$
q_{j} \geqq C, \quad \forall j \in \mathbb{N},
$$

then the spectrum of $H_{\alpha, \lambda=1}$ is a Cantor set with measure 0.

More recently, by quite different techniques, Y. Last get in $[19,20]$ that, for $0 \leqq \lambda \leqq 1$, the Lebesgue measure of the spectrum of $H_{\alpha, \lambda}$ is for a.e. $\alpha$ equal to $2|1-| \lambda||$. The case when $\lambda=1$ appears as a very important case and in this case Y. Last get (see Corollary 1.1 in [20]) that the spectrum is a zero measure Cantor set (i.e. closed, nowhere dense set, with no isolated points) for a.e. $\alpha$. More precisely the theorem is the following:

Theorem 1.2. If $\alpha$ is an irrational, for which there is a sequence of rationals $p_{n} / q_{n}$ obeying:

$$
\lim _{n \rightarrow \infty} q_{n}^{2}\left|\alpha-\frac{p_{n}}{q_{n}}\right|=0
$$


then for every $\lambda, \theta \in \mathbb{R}$ :

$$
|\sigma(\alpha, \lambda, \theta)|=2|1-| \lambda||
$$

where $|\cdot|$ denotes Lebesgue measure.

We now concentrate our study on the case when $\lambda=1$, where the measure of the spectrum is proved in Theorem 1.2 to be 0 . The proof of the theorem in this case is based on a careful study of the rational case and in this case the basic lemma is:

Lemma 1.3. If $p$ and $q$ are mutually prime (we then write $p \wedge q=1$ )

$$
\frac{(\sqrt{5}+1)}{q}<|\Sigma(p / q, 1)|<\frac{4 e}{q},
$$

with $e=\exp 1$.

A similar but weaker estimate of the lower bound was already obtained in [21]. This lemma is strongly related to a conjecture due to Thouless which says:

\section{Conjecture 1.4 .}

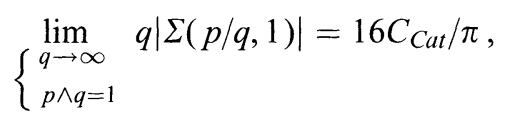

where $C_{C a t}$ is the so-called Catalan's constant

$$
C_{C a t}=\sum_{n \in \mathbb{N}}(-1)^{n}(2 n+1)^{-2}
$$

which appears also in Statistical Mechanics (see for example [30]) and is approximately equal to:

$$
C_{C a t} \approx 0.9159 \ldots
$$

This conjecture has been studied numerically and theoretically in $[27,29,25,26]$ (see also $[31]^{1}$ ). Thouless (sometimes with collaborators) gives in particular semiclassical arguments justifying the conjecture in the case $p=1$ and in the case $p=2 ; q$ odd. The proof is based on an analysis of the Green function but it is not completely clear to us if the proof is totally rigorous in the analysis of the remainders. Although complex WKB techniques are used in this approach, they are quite different of the approach we present here and more in the spirit of the recent approach by V. Buslaev and S. Fedotov [7,8 and 9].

The point of view of Y. Last and M. Wilkinson in [21] is more in the spirit of earlier works by M. Wilkinson [32] or [34] and uses semiclassical analysis in a microlocal spirit. We shall see later that all the spectrum is concentrated as $q \rightarrow \infty$ near 0 . The reason is that outside a fixed interval $]-\varepsilon,+\varepsilon[$ the spectrum is a union of bands which are exponentially small. This has been proved rigorously

\footnotetext{
1 We thank the referee for giving us this reference.
} 
(following the strategy of Wilkinson [32,34]) by B. Helffer and J. Sjöstrand in [12] who get in particular the following theorem:

Theorem 1.5. Let us assume $\lambda=1$. Let $\varepsilon_{0}>0$; there exists $C_{0}>0$, s.t. if $\alpha$ is in ]0, 1[ and admits the following expansion (finite or infinite) in continuous fraction:

$$
\alpha=\frac{1}{q_{1}+\frac{1}{q_{2}+\frac{1}{q_{3}+\cdots}}}
$$

with $q_{j} \in \mathbb{Z},\left|q_{1}\right| \geqq C_{0},\left|q_{J}\right| \geqq 1$ then

$$
\Sigma(\alpha, 1) \subset \bigcup_{N_{-} \leqq j \leqq N_{+}} J_{j},
$$

where the $J_{j}$ are closed disjoint intervals of length $\neq 0$, with $\partial J_{j} \subset \mathrm{Sp}(H) . J_{j+1}$ is on the right of $J_{j}$ and the distance $d_{j}$ between these two intervals is approximately:

$$
d_{j} \approx \frac{1}{\left|q_{1}\right|}
$$

$J_{0}$ is of length $2 \varepsilon_{0}+\mathcal{O}\left(1 / q_{1}\right)$ and centered at 0 . The other intervals are of length $\exp -C_{j} \cdot\left|q_{1}\right|$ with $C_{j} \approx 1$.

Here $a \approx b$ means that $a / b$ and $b / a$ are majorized by a constant which depends only on $\varepsilon_{0}$.

Finally $N_{+}=-N_{-} \approx\left|q_{1}\right|$.

Consequently, for any $\varepsilon_{0}>0$, the contribution in the total bandwidth which is outside ] $-\varepsilon_{0},+\varepsilon_{0}$ [ is exponentially small. Moreover, the proof gives a renormalization procedure. For $j \neq 0$, the spectrum in each interval is given, after an affine transformation, sending $J_{J}$ on essentially $[-2,2]$, by the spectrum of a suitable perturbation of the Harper's operator to which the preceding theorem can be again applied if $\left|q_{2}\right| \geqq C_{0}$. This perturbed Harper's operator is now an $h^{\prime}$-pseudodifferential operator with

$$
\frac{h^{\prime}}{2 \pi}=\frac{1}{q_{2}+\frac{1}{q_{3}+\frac{1}{q_{4}+\cdots}}} .
$$

In the rational case, the procedure stops after a finite number of steps. Note that if $\frac{h}{2 \pi}=\frac{1}{q_{1}}$, we get $h^{\prime}=0$, and the convention is that a $\left(h^{\prime}=0\right)$-pseudodifferential operator of symbol $p$ is then simply the operator of multiplication by $p$ on $L_{x, \xi}^{2}\left(\mathbb{R}^{2}\right)$, whose spectrum is simply the set $\left\{\lambda \in \mathbb{R}\right.$ s.t. $\exists(x, \xi) \in \mathbb{R}^{2}$ with $\left.\lambda=p(x, \xi)\right\}$.

In particular this says that the contribution in the total width is exponentially small outside ] $-\varepsilon_{0}, \varepsilon_{0}$ [. Hence we can not avoid the study of the spectrum of the Harper's operator near 0 which is much more difficult because 0 is a saddle point of the symbol of the operator:

$$
(x, \xi) \rightarrow \cos x+\cos \xi .
$$

This study was done at a heuristic level (and with some uncertainty) by Azbel [2] and the mathematical proof was given by B. Helffer and J. Sjöstrand in [14]. It is consequently natural to think that the machinery which was introduced in this paper can give a rigorous approach for the a priori easier problem consisting in measuring the total bandwidth. This problem is easier, in the sense that it appears 
as a one step problem and we shall not need the infinite sequence of approximate renormalizations used in [14] in order to prove the Cantor structure. Our main goal will be consequently to see what gives this strategy and we shall prove that it works at least in the two cases where reasonable mathematical arguments were already given. We consequently rigorously prove:

Theorem 1.6.

$$
\lim _{q \rightarrow \infty} q|\Sigma(1 / q, 1)|=(16 / \pi) C_{C a t}
$$

\section{Theorem 1.7.}

$$
\lim _{q \rightarrow \infty}(2 q+1)\left|\Sigma\left(\frac{2}{(2 q+1)}, 1\right)\right|=(16 / \pi) C_{C u t} .
$$

The second theorem will use also some techniques related to the semi-classical study near a rational [13].

This article is organized as follows:

Section 2 recalls some results of $[12,13$ and 14]. Section 3 is devoted to the special case when $\alpha=1 / q$. In Sect. 4 we shall treat the case when $\alpha=\frac{2}{2 q+1}=\frac{1}{q+\frac{1}{2}}$. The last section is devoted to the discussion of possible extensions or open problems.

\section{Semi-classical Analysis for Harper's Equation}

We now state what we have to know from the general theory in order to analyze our particular case and refer to [14]. No proof is recalled, but we collect all the statements proved in [14], permitting us to start a rigorous proof for the asymptotic behavior of the total bandwidth. We have already recalled Theorem 1.5 in this direction which gave us the information that this behavior was essentially obtained by restricting the study near 0 which corresponds to the saddle point of the symbol $(x, \xi) \rightarrow \cos x+\cos \xi$. The main result is the following (and gives a rigorous version of heuristic arguments due to Azbel [2]):

Theorem 2.1. There exists $\varepsilon_{0}>0, \varepsilon_{1}>0$ and $h_{0}$ such that, in the interval $\left[-\varepsilon_{0}, \varepsilon_{0}\right]$ and for $0<h<h_{0}, \mu$ is in the spectrum of the Harper's operator if and only if 0 is in the spectrum of a vectorvalued $h^{\prime}$-pseudodifferential operator $Q\left(x, h^{\prime} D_{x}, h, \mu^{\prime}\right)$ on $L^{2}\left(\mathbb{R} ; \mathbb{C}^{2}\right)$ whose symbol is a $2 \times 2$ matrix depending on a parameter $\mu^{\prime}$ and given by

$$
Q(x, \xi)=Q_{0}(x, \xi)+\mathcal{O}\left(\exp -\varepsilon_{1} / h\right)
$$

with

$$
Q_{0}(x, \xi)=\left(\begin{array}{ll}
b+\bar{a} \exp -i \xi & \bar{b}+a \exp i x \\
\bar{b}+a \exp -i x & b+\bar{a} \exp i \xi
\end{array}\right) .
$$

The parameter $h^{\prime}$ is related to $h$ by the relation

$$
\frac{2 \pi}{h^{\prime}}=\frac{h}{2 \pi} \bmod \mathbb{Z} \text {. }
$$

The parameter $\mu^{\prime}$ is related to the spectral parameter $\mu$ by $\mu^{\prime}=f(\mu, h)$, where $f$ is the realization of a formal real valued symbol, i.e. admits in $\left[-2 \varepsilon_{0}, 2 \varepsilon_{0}\right]$ the 
following expansion:

$$
\begin{aligned}
f(\mu, h) & \equiv f_{0}(\mu)+h f_{1}(\mu)+\cdots, \\
f_{0}(0) & =0 ; \quad f_{0}^{\prime}(0)=1 .
\end{aligned}
$$

The parameters $a$ and $b$ are given $b y$

$$
\begin{aligned}
& b=(2 \pi)^{-1 / 2} \Gamma\left(\frac{1}{2}-i \frac{\mu^{\prime}}{h}\right) \exp \left[i \frac{\mu^{\prime}}{h} \ln \left(\frac{1}{h}\right)+\pi \frac{\mu^{\prime}}{2 h}+i \frac{g\left(\mu^{\prime}, h\right)}{h}\right], \\
& a=(2 \pi)^{-1 / 2} \Gamma\left(\frac{1}{2}-i \frac{\mu^{\prime}}{h}\right) \exp \left[i \frac{\mu^{\prime}}{h} \ln \left(\frac{1}{h}\right)-\pi \frac{\mu^{\prime}}{2 h}+i \frac{g\left(\mu^{\prime}, h\right)}{h}-i \frac{\pi}{2}\right],
\end{aligned}
$$

where $\Gamma$ is the standard gamma function, $g$ is a classical analytic symbol of order $\leqq 0$ which can be assumed to be real valued.

Remark 2.2. The operator $Q$ above is unfortunately not selfadjoint and it is quite useful in order to use perturbation theory to come back to a selfadjoint theory. That this is possible is of course not strange if we recall that our initial problem was selfadjoint. The proof given in [14] keeps actually a "memory" of this property by giving an explicit way of selfadjointization. More precisely, the proof gives also the existence of a family of operators $P_{1}\left(x, h^{\prime} D_{x}, \theta, h, \mu^{\prime}\right)$ of the same type of $Q$ such that:

$$
P_{1}^{\star} Q=Q^{\star} P_{1}
$$

and

$$
\left.P_{1}(x, \xi, \theta)=P_{1,0}(x, \xi, \theta)+\mathcal{O}\left(\exp -\varepsilon_{1} / h\right)\right)
$$

with

$$
P_{1,0}(x, \xi, \theta)=\left(\begin{array}{ll}
b^{\prime}+\bar{a}^{\prime} \exp -i \xi & \bar{b}^{\prime}+a^{\prime} \exp i x \\
\bar{b}^{\prime}+a^{\prime} \exp -i x & b+\bar{a}^{\prime} \exp i \xi
\end{array}\right),
$$

with

$$
b^{\prime}=b \exp i \theta ; \quad a^{\prime}=a \exp i \theta .
$$

Similarly, it is possible to define another family of operators $P_{2}$ of the same type such that:

$$
Q P_{2}^{\star}=P_{2} Q^{\star}
$$

Let us now establish useful relations. We get for $\mu^{\prime} \in \mathbb{R}$ the following relations:

$$
\begin{gathered}
|a|^{2}+|b|^{2}=1, \\
\arg (b)-\arg (a)=\pi / 2, \\
a \bar{b}=-\bar{a} b=-i|a| \cdot|b|, \\
|b|=\exp +\pi \frac{\mu^{\prime}}{2 h}\left(\exp \pi \frac{\mu^{\prime}}{h}+\exp -\pi \frac{\mu^{\prime}}{h}\right)^{-1 / 2}, \\
|a|=\exp -\pi \frac{\mu^{\prime}}{2 h}\left(\exp \pi \frac{\mu^{\prime}}{h}+\exp -\pi \frac{\mu^{\prime}}{h}\right)^{-1 / 2}, \\
|a||b|=1 /\left(2 \cosh \left(\pi \frac{\mu^{\prime}}{h}\right)\right), \quad|a|^{2}-|b|^{2}=-\tanh \left(\pi \frac{\mu^{\prime}}{h}\right) .
\end{gathered}
$$


The determinant of the matrix $Q_{0}$ has the following form, as a function of $a, b$ satisfying the conditions $(2.6),(2.7)$ :

$$
\operatorname{det} Q_{0}(x, \xi)=2 i[\sin (2 \arg b)+|a||b|(\cos \xi+\cos x)] .
$$

Similarly, the determinant of the matrix $P_{1,0}$ is given by

$$
\operatorname{det} P_{1,0}(x, \xi, \theta)=2 i[\sin (2 \arg b+2 \theta)+|a||b|(\cos \xi+\cos x)] .
$$

Let us now discuss more precisely from where the function $f$ comes. It is important to remark that the above theorem is not only proved for the Harper's equation, but also for small perturbations of this operator. This point was crucial in the renormalization analysis (see [14]) or in the applications to the study of the Schrödinger operator with magnetic field [12]. This is also important in the extensions which will be sketched in the last section where we discussed the results of Last and Wilkinson [21] using the rigorous techniques developed in [13]. The role of $f$ will be clear if we recall the following theorem (Theorem b.1. in [14]):

Theorem 2.3. Let $P\left(x, h D_{x}, h\right)$ be a formal classical analytic pseudodifferential operator, of order 0 , formally selfadjoint, whose symbol is defined in a neighborhood of $(0,0)$. Let $p$ be the principal symbol, and assume that $p$ has a nondegenerate saddle point at $(0,0)$ with critical value 0 . Then there is a real-valued analytic symbol: $\mu \rightarrow f(\mu, h)$ defined for $\mu$ in a neighborhood of 0 , and a formal unitary analytic Fourier integral operator, whose associated canonical transformation (in the classical sense) is defined in a neighborhood of $(0,0)$, and maps this point onto itself, such that

$$
U^{\star} f(P, h) U=\frac{1}{2}\left(x h D_{x}+h D_{x} x\right)
$$

It is not crucial for the reader to understand (at the first reading) for this paper what is the analytic machinery (which is only used in the proof of the theorems we are referring to, but permits to work modulo exponentially small terms). It is impossible to give more details here in this short presentation of some of the results of [14], but let us just add the point that this last theorem was crucial in the precise construction of WKB solutions near the saddle point.

More important for further generalizations are the following remarks coming from the proof:

Remark 2.4. One can concretely find the first term $f_{0}$ of $f$. The function $\mu \rightarrow f_{0}(\mu)$ is determined by the condition that the complex period $T(\mu)$ of the hamiltonian flow $H_{p_{0}}$ on the energy level $p_{0}(x, \xi)=\mu$ becomes, by replacing $p_{0}$ by $f_{0}\left(p_{0}\right)$ and near the energy corresponding to the saddle point, independent of the energy and equal to $T_{0}=2 i \pi$ which is the complex period of the hamiltonian flow attached to the model $(x, \xi) \rightarrow x \cdot \xi$. In the case when $p_{0}(x, \xi)=\cos x+\cos \xi$, we observe that the Taylor expansion at the order 2 is given. For example the point $(0, \pi)$ is given by $\left(-x^{2}+(\xi-\pi)^{2}\right) / 2$ and this explains why we have condition (2.5) in the case of the Harper's operator.

More precisely, as was indicated to us by S. Fedotov, we have in this case the following formula:

$$
f_{0}(\mu)=\frac{1}{\pi} \operatorname{sign}(\mu) S(\mu),
$$


where $S(\mu)$ is the tunneling parameter:

$$
\begin{aligned}
& \text { if } \mu \geqq 0, S(\mu)=\int_{a}^{2 \pi-a} \cosh ^{-1}(\mu-\cos x) d x, \\
& \text { with } \mu=1+\cos a, 0 \leqq a \leqq \pi ; \\
& \text { if } \mu \leqq 0, S(\mu)=S(-\mu)=\int_{b}^{4 \pi-b} \cosh ^{-1}(-\mu+\cos x) d x, \\
& \text { with } \mu=-1+\cos b, \pi \leqq b \leqq 2 \pi .
\end{aligned}
$$

The proof is classical (at least in the case of hamiltonian mechanics in the classical region) and is based on the property (see e.g. [14] Appendix b):

$$
f_{0}^{\prime}(\mu)=\frac{T(\mu)}{T_{0}} .
$$

The asymptotic behavior of the total bandwidth depends actually only on $f$ through $f_{0}^{\prime}(\mathbf{0})$

Remark 2.5. The function $g$ contains a global information on the area of domains delimited by the energy surfaces near the critical one but this will not appear in the main term of our asymptotics.

Remark 2.6. The new parameter $h^{\prime}$ which is in this context given by (2.3) appears in the construction of two operators on $L^{2}(\mathbb{R}) \tau$ and $\tau^{\star}$ commuting with the Harper's operator and satisfying the following commutation relations:

$$
\begin{aligned}
\tau H & =H \tau, \quad \tau^{\star} H=H \tau^{\star}, \\
\tau \tau^{\star} & =\left(\exp -i h^{\prime}\right) \tau^{\star} \tau .
\end{aligned}
$$

In this particular case, we have actually:

$$
(\tau u)(x)=u(x-2 \pi), \quad\left(\tau^{\star} u\right)(x)=\exp \frac{2 i \pi x}{h} u(x)
$$

which explains (2.3).

We recall finally some properties (cf. [14] and (2.7), (2.8)) of $Q$ which are easily and directly verified for $Q_{0}$ :

$$
\begin{aligned}
Q(-\xi, x) & =\left(\begin{array}{ll}
0 & 1 \\
1 & 0
\end{array}\right) Q^{\star}(x, \xi), \\
\bar{Q}(\xi, x) & =Q(x, \xi)\left(\begin{array}{ll}
0 & 1 \\
1 & 0
\end{array}\right), \\
Q(-x,-\xi) & =\left(\begin{array}{ll}
0 & 1 \\
1 & 0
\end{array}\right) Q(x, \xi)\left(\begin{array}{ll}
0 & 1 \\
1 & 0
\end{array}\right) .
\end{aligned}
$$

Two particular cases are analyzed in the next sections. If

$$
\alpha=\frac{h}{2 \pi}=1 / q
$$


then we get the special case

$$
h^{\prime}=0
$$

and Theorem 2.1 will reduce our study to the study of a family of $2 \times 2$-matrices. This will be analyzed in Sect. 3 . The second case corresponds to the assumption:

$$
\alpha=\frac{h}{2 \pi}=\frac{1}{q+\frac{1}{2}}
$$

which corresponds to:

$$
h^{\prime} / 2 \pi=1 / 2 .
$$

A Floquet theory will permit us to reduce again to the study of a family of $4 \times 4$-matrices. This will be presented in Sect. 4 .

\section{The Case $\alpha=1 / q$}

Let us apply Theorem 2.1. Here $h^{\prime}=0$ and, according to our conventions, the $h^{\prime}$-pseudodifferential operators have to be considered as multiplication operators defined on $L^{2}\left(\mathbb{R}_{x, \xi}^{2}\right)$. So the theorem says in our particular case that there exists $\varepsilon_{0}>0$ and $h_{0}$ such that, in the interval $\left[-\varepsilon_{0}, \varepsilon_{0}\right]$ and for $0<h<h_{0}, \mu$ is in the spectrum of the Harper's operator if and only if there exists $(x, \xi)$ s.t. $Q\left(x, \xi, h, \mu^{\prime}\right)$ is not injective. So we get finally the following transposition of Theorem 2.1:

Proposition 3.1. There exists $\varepsilon_{0}>0$ and $h_{0}$ such that, in the interval $\left[-\varepsilon_{0}, \varepsilon\right]$ and for $0<h<h_{0}, \mu$ is in the spectrum of the Harper's operator if and only if

$$
\text { there exists }(x, \xi) \text { s.t } \operatorname{det} Q\left(x, \xi, h, \mu^{\prime}\right)=0 \text { with } \mu^{\prime}=f(\mu, h) \text {. }
$$

Moreover we have

$$
\operatorname{det} Q=\operatorname{det} Q_{0}+\mathcal{O}\left(\exp -\varepsilon_{1} / h\right)
$$

with

$$
\operatorname{det} Q_{0}(x, \xi)=\left(i / \cosh \left(\pi \mu^{\prime} / h\right)\right)\left[2 \cosh \left(\pi \mu^{\prime} / h\right) \sin (2 \arg b)+\cos \xi+\cos x\right],
$$

and there exists $P_{1}(x, \xi, \theta) \in C^{\infty}\left(\mathbb{R}^{2} ; M_{2}(\mathbb{C})\right)$ s.t. $P_{1}^{\star} Q$ is selfadjoint for all $\theta$ and

$$
\begin{aligned}
\operatorname{det} P_{1}(x, \xi)= & \left(i / \cosh \left(\pi \mu^{\prime} / h\right)\right)\left[2 \cosh \left(\pi \mu^{\prime} / h\right) \sin (2(\arg b+\theta))\right. \\
& +\cos \xi+\cos x]+\mathcal{O}\left(\exp -\varepsilon_{1} / h\right)
\end{aligned}
$$

for some $\varepsilon_{1}>0$.

Let us denote by $\tilde{\sigma}_{h}$ the image by $f$ of $\sigma_{h}$. This is not defined outside $\left[-\varepsilon_{0}, \varepsilon_{0}\right]$ but we have seen that $\left[-\varepsilon_{0}, \varepsilon_{0}\right]$ is the interesting region where the spectrum is concentrated as $h \rightarrow 0$. We consider first

$$
\frac{1}{h}\left|\tilde{\sigma}_{h} \cap\left(\left[-c,-\varepsilon_{2} h\right] \cup\left[\varepsilon_{2} h, c\right]\right)\right|,
$$

where $c>0$ is fixed sufficiently small such that

$$
\left.f^{-1}([-c,+c]) \subset\right]-\varepsilon_{0}, \varepsilon_{0}\left[, \quad \pi c \leqq \varepsilon_{1} / 2\right.
$$


and $\varepsilon_{2}>0$ will be chosen later arbitrarily small. We choose now

$$
\theta=\left(\frac{\pi}{4}-\arg b\right) \text {. }
$$

This implies the existence of $C_{1}\left(\varepsilon_{2}\right)>0$ such that, for $\mu^{\prime} \in I\left(\varepsilon_{2}, h\right)=\left[-c,-\varepsilon_{2} h\right] \cup$ $\left[+\varepsilon_{2} h,+c\right]$,

$$
\left|\operatorname{det} P_{1}(x, \xi)\right| \geqq C_{1}\left(\varepsilon_{2}\right)>0 \text {. }
$$

Let us first sketch an heuristic proof. (The correct proof will be given later.) If we forget the remainder term in (3.1)-(3.2) we get the condition

$$
\cosh \left(\pi \mu^{\prime} / h\right) \sin (2 \arg b) \in[-1,+1] \text {. }
$$

In the interval $I\left(\varepsilon_{2}, h\right)$ the variation of $\arg b$ is much larger in comparison with the variation of $1 / \cosh \pi \frac{\mu^{\prime}}{h}$. We have indeed (see (5.13) in [14]):

$$
\left|\partial_{\mu^{\prime}} \arg b\right|=h^{-1} \ln \left(1 /\left(h+\left|\mu^{\prime}\right|\right)\right)+\mathcal{O}(1 / h)
$$

and

$$
\left|\partial_{\mu^{\prime}}\left(1 / \cosh \pi \frac{\mu^{\prime}}{h}\right)\right| \leqq C h^{-1}\left(\cosh \pi \frac{\mu^{\prime}}{h}\right)^{-1} .
$$

In particular we observe that the quotient satisfies in the interval $I$,

$$
\left|\partial_{\mu^{\prime}} \arg b\right| /\left|\partial_{\mu^{\prime}}\left(1 / \cosh \pi \frac{\mu^{\prime}}{h}\right)\right| \geqq(1 / C) \ln (1 / h) .
$$

We compute then approximately the length of the spectrum contained in $\left[\mu_{1}^{\prime \prime} h, \mu_{2}^{\prime \prime} h\right]$ by writing that

$$
\frac{\left|\tilde{\sigma}_{h} \cap\left[\mu_{1}^{\prime \prime} h, \mu_{2}^{\prime \prime} h\right]\right|}{h\left(\mu_{2}^{\prime \prime}-\mu_{1}^{\prime \prime}\right)} \approx \frac{\arcsin \left(1 / \cosh \pi \frac{\mu_{0}^{\prime}}{h}\right)}{\frac{\pi}{2}},
$$

for some $\mu_{0}^{\prime}$ in the interval, and get after summation (using the Riemann approximation of the integral)

$$
\left|\tilde{\sigma}_{h}\right|_{\left[\varepsilon_{2} h, c\right]}=\frac{4 h}{\pi}\left(\int_{\varepsilon_{2}}^{c / h} \arcsin (1 / \cosh \pi s) d s\right) .
$$

Let us now transform this heuristic proof into a rigorous one. The first step is the following lemma:

Lemma 3.2. For any $\varepsilon_{2}>0$, there exists $C_{2}>0$ such that

$$
\begin{aligned}
& \left\{\mu^{\prime} \in I ;\left|\cosh \left(\frac{\pi \mu^{\prime}}{h}\right) \sin (2 \arg b)\right| \leqq 1-C_{2} \exp -\frac{\varepsilon_{1}}{2 h}\right\} \subset \tilde{\sigma}_{h} \cap I ; \\
& \tilde{\sigma}_{h} \cap I \subset\left\{\mu^{\prime} \in I ;\left|\cosh \left(\frac{\pi \mu^{\prime}}{h}\right) \sin (2 \arg b)\right| \leqq 1+C_{2} \exp -\frac{\varepsilon_{1}}{2 h}\right\} .
\end{aligned}
$$

Proof. The second inclusion is an immediate consequence of (3.2) if we choose $C_{2}$ large enough. Let us prove the first one and this is the point where the mysterious procedure of selfadjointization appears. We observe that:

$$
\begin{aligned}
\cosh \left(\frac{\pi \mu^{\prime}}{h}\right)^{2} \operatorname{det}\left(P_{1}^{\star} Q\right)= & {\left[\cosh \left(\frac{\pi \mu^{\prime}}{h}\right) \sin (2 \arg b)+\cos x+\cos \xi+\mathcal{O}\left(\exp -\frac{\varepsilon_{1}}{2 h}\right)\right] } \\
& \times\left[\cosh \left(\frac{\pi \mu^{\prime}}{h}\right)+\cos x+\cos \xi+\mathcal{O}\left(\exp -\frac{\varepsilon_{1}}{2 h}\right)\right]+\mathcal{O}\left(\exp -\frac{\varepsilon_{1}}{2 h}\right),
\end{aligned}
$$


where all the remainder terms in the right-hand side are now real. The term in the second bracket is strictly positive for $h$ small enough:

$$
\left[\cosh \left(\frac{\pi \mu^{\prime}}{h}\right)+\cos x+\cos \xi\right]+\mathcal{O}\left(\exp -\frac{\varepsilon_{1}}{2 h}\right) \geqq 2 \cosh \left(\pi \varepsilon_{2}\right)-2>0 \text {. }
$$

If $C_{2}$ is chosen sufficiently large, then

$$
\operatorname{det}\left(P_{1}^{\star} Q\right)(0,0)>0 \text { and } \operatorname{det}\left(P_{1}^{\star} Q\right)(\pi, \pi)<0 \text {. }
$$

Hence there exists $(x, \xi)$ such that $\operatorname{det}\left(P_{1}^{\star} Q\right)(x, \xi)=0$ and using (3.5), we finally get

$$
\operatorname{det}(Q)(x, \xi)=0,
$$

that is $\mu^{\prime} \in \tilde{\sigma}_{h}$. The lemma is proved.

We can now write more precisely the argument which was sketched above. If $\mu_{1}^{\prime \prime}$ and $\mu_{2}^{\prime \prime}$ satisfy

$$
\varepsilon_{2} h \leqq \mu_{1}^{\prime \prime} h<\mu_{2}^{\prime \prime} h \leqq c,
$$

then (3.7) and (3.8) imply the following inclusions:

$$
\tilde{\sigma}_{h} \cap\left[\mu_{1}^{\prime \prime} h, \mu_{2}^{\prime \prime} h\right] \subset \bigcup_{\substack{\arg b\left(\mu_{0}^{\prime}\right) \equiv 0\left[\frac{\pi}{2}\right] \\ \mu_{1}^{\prime \prime} h \leqq \mu_{0}^{\prime} \leqq \mu_{2}^{\prime \prime} h}} I_{\max }\left(\mu_{0}^{\prime}\right)
$$

where $I_{\max }\left(\mu_{0}^{\prime}\right)$ is an interval centered at $\mu_{0}^{\prime}$ and of length

$$
\left|I_{\max }\left(\mu_{0}^{\prime}\right)\right|=\left(2 \arcsin \frac{1}{\cosh \left(\pi \mu_{1}^{\prime \prime}\right)}\right)\left(\frac{h}{-\ln \left(h+\left|\mu_{2}^{\prime \prime}\right| h\right)-C_{4}}\right),
$$

where $C_{4}$ is chosen sufficiently large. Similarly, we have:

$$
\bigcup_{\substack{\arg b\left(\mu_{0}^{\prime}\right) \equiv 0\left[\frac{\pi}{2}\right] \\ I_{\min }\left(\mu_{0}^{\prime}\right) \subset\left[\mu_{1}^{\prime} h, \mu_{2}^{\prime \prime} h\right]}} I_{\min }\left(\mu_{0}^{\prime}\right) \subset \tilde{\sigma}_{h} \cap\left[\mu_{1}^{\prime \prime} h, \mu_{2}^{\prime \prime} h\right],
$$

where $I_{\min }\left(\mu_{0}^{\prime}\right)$ is an interval centered at $\mu_{0}^{\prime}$ and of length

$$
\left|I_{\min }\left(\mu_{0}^{\prime}\right)\right|=\left(2 \arcsin \frac{1}{\cosh \left(\pi \mu_{2}^{\prime \prime}\right)}\right)\left(\frac{h}{-\ln \left(h+\left|\mu_{1}^{\prime \prime}\right| h\right)+C_{4}}\right),
$$

where $C_{4}$ is chosen sufficiently large.

We have also the following estimate for the number $v\left(\left[\mu_{1}^{\prime \prime} h, \mu_{2}^{\prime \prime} h\right]\right)$ of $\mu_{0}^{\prime}$ such that $\arg b\left(\mu_{0}^{\prime}\right) \equiv 0\left[\frac{\pi}{2}\right]$ and $\mu_{0}^{\prime} \in\left[\mu_{1}^{\prime \prime} h, \mu_{2}^{\prime \prime} h\right]$ :

$$
\begin{aligned}
& \pi^{-1}\left(\mu_{2}^{\prime \prime}-\mu_{1}^{\prime \prime}\right)\left(-\ln \left(h+\left|\mu_{2}^{\prime \prime}\right| h\right)-C_{4}\right) \leqq v\left(\left[\mu_{1}^{\prime \prime} h, \mu_{2}^{\prime \prime} h\right]\right), \\
& v\left(\left[\mu_{1}^{\prime \prime} h, \mu_{2}^{\prime \prime} h\right]\right) \leqq \pi^{-1}\left(\mu_{2}^{\prime \prime}-\mu_{1}^{\prime \prime}\right)\left(-\ln \left(h+\left|\mu_{1}^{\prime \prime}\right| h\right)+C_{4}\right) .
\end{aligned}
$$

Let us first compute the contribution corresponding to $\tilde{\sigma}_{h} \cap[N h, c]$ with $N$ large. Using (3.8), (3.11) and (3.12) and a decomposition by intervals $[n h,(n+1) h]$, we 
get the following majoration, for $N \geqq N_{0}$ with $N_{0}$ large:

$$
\begin{aligned}
\frac{1}{h}\left|\tilde{\sigma}_{h} \cap[N h, c]\right| \leqq & \sum_{n \geqq N} \frac{2}{\pi} \arcsin \frac{1}{\cosh \pi n}\left(\frac{1}{\left(-\ln (h(n+2))-C_{4}\right)}\right) \\
& \times\left(-\ln h(n+1)+C_{4}\right) \\
\leqq & C \sum_{n=N}^{\infty} \frac{1}{\cosh \pi n} .
\end{aligned}
$$

This last quantity tends to 0 . We have consequently obtained that for any $\eta>0$ there exists $N_{1}$ and $h_{1}$ such that:

$$
\frac{1}{h}\left|\tilde{\sigma}_{h} \cap\left[N_{1} h, c\right]\right| \leqq \eta, \quad \forall h \leqq h_{1} .
$$

We now consider the spectrum $\tilde{\sigma}_{h}$ in the interval $\left[\varepsilon_{2} h, N h\right]$ for fixed $N$. We decompose this interval into $M(h)$ intervals $U_{k}(h)(k=1, \ldots, M(h))$ of length $h \delta(h) \approx h\left(\ln \frac{1}{h}\right)^{-1 / 2}$. With this choice of length for the interval $U_{k}$, we have (with $N$ fixed):

$$
v\left(U_{k}\right)=\pi(-\ln h)^{1 / 2}\left(1+o_{N}(1)\right)
$$

and we observe that if we only count the number $v^{\prime}\left(U_{k}\right)$ of the $\mu_{0}^{\prime}$ such that $\arg b\left(\mu_{0}^{\prime}\right) \equiv 0\left[\frac{\pi}{2}\right]$ and $I_{\min } \subset U_{k}$, then we have:

$$
v\left(U_{k}\right)-2 \leqq v^{\prime}\left(U_{k}\right) \leqq v\left(U_{k}\right) .
$$

In particular, independently of $k$ (but depending on $N$ ), we have:

$$
v\left(U_{k}\right) / v^{\prime}\left(U_{k}\right) \rightarrow 1, \quad \text { as } h \rightarrow 0 .
$$

This last point is actually only useful for the minoration. Let us first consider the majoration where we can divide simply the interval in equal intervals. We get:

$$
\frac{1}{h}\left|\tilde{\sigma}_{h} \cap\left[\varepsilon_{2} h, N h\right]\right| \leqq \sum_{n=1}^{M(h)} \delta(h) \frac{2}{\pi} \arcsin \frac{1}{\cosh \pi\left(\varepsilon_{2}+n \delta(h)\right)} \cdot A(n, h),
$$

where

$$
A(n, h)=\frac{\left(-\ln \left(h+\left(\varepsilon_{2}+n \delta(h)\right) h\right)+C_{4}\right)}{\left(-\ln \left(h+\left(\varepsilon_{2}+(n+1) \delta(h)\right) h\right)-C_{4}\right)} .
$$

We now observe that this last term tends to 1 as $h$ tend to 0 uniformly with respect to $n$ satisfying $0 \leqq n \leqq M(h)$. We consequently obtain:

$$
\frac{1}{h}\left|\tilde{\sigma}_{h} \cap\left[\varepsilon_{2} h, N h\right]\right| \leqq \sum_{n=1}^{M(h)} \delta(h) \frac{2}{\pi} \arcsin \frac{1}{\cosh \pi\left(\varepsilon_{2}+n \delta(h)\right)} \cdot(1+o(1))
$$

and the right-hand side tends to $\frac{2}{\pi} \int_{\varepsilon_{2}}^{N} \arcsin (1 / \cosh \pi s) d s$ as $h \rightarrow 0$.

Modulo the considerations around (3.16), we proceed on the same way for the minoration and finally we have proved:

\section{Lemma 3.3.}

$$
\frac{\left|\tilde{\sigma}_{h} \cap\left[\varepsilon_{2} h, c\right]\right|}{h} \rightarrow \frac{2}{\pi} \int_{\varepsilon_{2}}^{\infty} \arcsin (1 / \cosh \pi s) d s .
$$




\section{Similarly}

$$
\frac{\left|\tilde{\sigma}_{h} \cap\left[-c,-\varepsilon_{2} h\right]\right|}{h} \rightarrow \frac{2}{\pi} \int_{-\varepsilon_{2}}^{-\infty} \arcsin (1 / \cosh \pi s) d s
$$

It remains now to come back to the $\mu$ variable. Using (2.5), we get the existence of a constant $C$. such that for any $\varepsilon_{3}$ s.t. $0<\varepsilon_{3}<c$ and for any $\varepsilon_{2}>0$ :

$$
\begin{aligned}
&\left|\tilde{\sigma}_{h} \cap\left[\varepsilon_{2} h, \varepsilon_{3}\right]\right|\left(1-C\left(h+\varepsilon_{3}\right) \mid\right. \leqq\left|f^{-1}\left(\tilde{\sigma}_{h} \cap\left[\varepsilon_{2} h, \varepsilon_{3}\right]\right)\right| \\
& \leqq\left|\tilde{\sigma}_{h} \cap\left[\varepsilon_{2} h, \varepsilon_{3}\right]\right|\left(1+C\left(h+\varepsilon_{3}\right) \mid,\right. \\
&\left|f^{-1}\left(\tilde{\sigma}_{h} \cap\left[-\varepsilon_{2} h, \varepsilon_{3} h\right]\right)\right| \leqq C \varepsilon_{2} h
\end{aligned}
$$

and

$$
\left|f^{-1}\left(\tilde{\sigma}_{h} \cap\left[\varepsilon_{3}, c\right]\right)\right| \leqq C\left(\varepsilon_{3}\right) \exp -\left(C\left(\varepsilon_{3}\right) / h\right),
$$

with $C\left(\varepsilon_{3}\right)>0$. Let us also recall that:

$$
f^{-1}(0)=\mathcal{O}(h)
$$

and

$$
\partial f / \partial \mu^{\prime}=1+\mathcal{O}(h+c) \text { on }[-c, c] .
$$

We then get by combining the different estimates:

$$
\frac{\left|\sigma_{h}\right|}{h} \rightarrow_{h \rightarrow 0} \frac{4}{\pi} \int_{-\infty}^{0} \arcsin (1 / \cosh \pi s) d s
$$

Let us compute the r.h.s. differently:

$$
\begin{aligned}
& \frac{4}{\pi} \int_{-\infty}^{0} \arcsin (1 / \cosh \pi s) d s \\
& \quad=\frac{4}{\pi} \int_{-\infty}^{0} \arcsin \left(2 \exp \pi s /(1+\exp 2 \pi s)(\pi \exp \pi s)^{-1} d \exp \pi s\right. \\
& \quad=\frac{4}{\pi^{2}} \int_{0}^{1} \arcsin \left(2 t /\left(1+t^{2}\right)\right) d t / t \\
& =\frac{8}{\pi^{2}} \int_{0}^{1} \arctan t d t / t \\
& \quad=\frac{8}{\pi^{2}} C_{\text {cat }} .
\end{aligned}
$$

We now take $h=2 \pi \alpha=2 \pi / q$ and finally get:

$$
q\left|\sigma_{2 \pi / q}\right| \rightarrow \frac{16}{\pi} C_{c a t},
$$

which corresponds to the result of Thouless [29]. 


\section{The Case $\alpha=2 /(2 q+1)$}

As we have already observed we have to analyze the $(\pi)$-pseudodifferential operator whose symbol is a very small perturbation of the $2 \times 2$ matrix valued function:

$$
(x, \xi) \rightarrow Q_{0}(x, \xi) .
$$

We follow the strategy of ([13]) which was already introduced by other authors (see the survey of J. Bellissard [5] for references and also [32,24]...). We forget for the moment the remainder. The study of a $\pi$-pseudodifferential operator with $2 \pi$ periodic symbol in $(x, \xi)$ is reduced to the study of the set described by the eigenvalues of the $4 \times 4$ matrix:

$$
Q_{0}(x, \xi)=\left(\begin{array}{ll}
b I+\bar{a} \exp -i \xi J^{-1} & \bar{b} I+a K \exp i x \\
\bar{b} I+a K^{-1} \exp -i x & b I+\bar{a} J \exp i \xi
\end{array}\right),
$$

where

$$
I=\left(\begin{array}{cc}
1 & 0 \\
0 & 1
\end{array}\right), \quad J=\left(\begin{array}{cc}
1 & 0 \\
0 & -1
\end{array}\right) \quad \text { and } \quad K=\left(\begin{array}{ll}
0 & 1 \\
1 & 0
\end{array}\right) .
$$

We cannot avoid for the moment the explicit computation of the determinant but let us first try to look at the problem in a more general context. We observe that our matrices $J$ and $K$ satisfy the following relations:

$$
J^{2}=I, \quad K^{2}=I, \quad J K=-K J .
$$

As in [13], we can more generally consider the two $q \times q$ matrices $J$ and $K$ attached to $\frac{1}{q}$ which satisfies the following relations:

$$
J^{q}=I, \quad K^{q}=I, \quad J K=\omega K J,
$$

with $\omega=\exp \frac{2 i \pi}{q}$. We can take for example:

$$
J=\left(\begin{array}{ccccc}
1 & 0 & & & \\
0 & \omega & 0 & & \\
& 0 & . & & \\
& & & . & 0 \\
& & & 0 & \omega^{q-1}
\end{array}\right) ; \quad K=\left(\begin{array}{ccccc}
0 & 0 & . & 0 & 1 \\
1 & 0 & . & . & 0 \\
0 & 1 & . & . & . \\
. & . & . & . & 0 \\
0 & . & 0 & 1 & 0
\end{array}\right) .
$$

We consider an irreducible rational $\frac{p}{q}$ and consider the corresponding family $2 q \times 2 q$-matrix $Q_{0, \frac{p}{q}}(x, \xi)$ associated to $(J, K)$ by the following formula (which extends the formula (4.1)):

$$
Q_{0, \frac{p}{q}}(x, \xi)=\left(\begin{array}{ll}
b I+\bar{a} \exp -i \xi J^{-p} & \bar{b} I+a K \exp i x \\
\bar{b} I+a K^{-1} \exp -i x & b I+\bar{a} J^{p} \exp i \xi
\end{array}\right) .
$$

We are expecting that the determinant of this matrix has a very particular structure as was observed in the case when $q=1$. We follow here, in a particular case, the study of symmetries realized in [14] for the so-called "type 2" operators. We 
first consider the commutation of this matrix with $\mathscr{J}=\left(\begin{array}{ll}J & 0 \\ 0 & J\end{array}\right)$ and observe the following relation:

$$
\mathscr{J} Q_{0, \frac{p}{q}}(x, \xi)=Q_{0, \frac{p}{q}}\left(x+\frac{2 \pi}{q}, \xi\right) \mathscr{J}
$$

Similarly we get also:

$$
\mathscr{K} Q_{0, \frac{p}{q}}(x, \xi)=Q_{0, \frac{p}{q}}\left(x, \xi-\frac{2 \pi p}{q}\right) \mathscr{K} .
$$

As an immediate consequence (using the property that $p$ and $q$ are mutually prime), we get that:

$$
(x, \xi) \rightarrow \operatorname{det}\left(Q_{0, \frac{p}{q}}(x, \xi)\right)
$$

is $2 \pi / q$ periodic with respect to $x$ and $\xi$. We note also that this determinant is a trigonometric polynomial with respect to the two variables $x$ and $\xi$. We have still to consider other symmetries. We first observe by considering the transpose that:

$$
{ }^{t} Q_{0, \frac{p}{q}}(x, \xi)=Q_{0, \frac{p}{q}}(-x, \xi)
$$

which implies:

$$
\operatorname{det} Q_{0, \frac{p}{q}}(x, \xi)=\operatorname{det} Q_{0, \frac{p}{q}}(-x, \xi) .
$$

As in [13], we introduce the finite Fourier transform $U_{p}$ which is the $q \times q$ unitary matrix

$$
\left(U_{p}\right)_{j k}=\frac{1}{\sqrt{q}} \omega^{p(j-1)(k-1)}, \quad \text { for } j=1, \ldots, q ; k=1, \ldots, q,
$$

with the following properties:

$$
U_{p}^{\star} J^{p} U_{p}=K ; \quad U_{p}^{\star} K U_{p}=J^{-p} .
$$

As before for $J$ and $K$, we extend $U_{p}$ into $\mathscr{U}_{p}=\left(\begin{array}{cc}U_{p} & 0 \\ 0 & U_{p}\end{array}\right)$.

Considering $\mathscr{U}_{p}^{\star} \bar{Q} \mathscr{U}_{p}$, we deduce also:

$$
\overline{\operatorname{det} Q_{0, \frac{p}{q}}(x, \xi)}=(-1)^{q} \operatorname{det} Q_{0, \frac{p}{q}}(\xi, x) .
$$

Using the other relation we then get also:

$$
\operatorname{det} Q_{0, \frac{p}{q}}(x,-\xi)=\operatorname{det} Q_{0, \frac{p}{q}}(x, \xi) .
$$

It is also interesting to look a priori for the dependence on $a$ and $b$. We observe for example that:

$$
\operatorname{det} Q_{0, \frac{p}{q}}(-x,-\xi ; a, b)=(-1)^{q} \operatorname{det} Q_{0, \frac{p}{q}}(x, \xi ; \bar{a}, \bar{b}) .
$$

We conclude from these arguments that:

$$
\begin{aligned}
\operatorname{det} Q_{0}(x, \xi)= & i^{q}\left[P_{0}(a, \bar{a}, b, \bar{b})+P_{1}(a, \bar{a}, b, \bar{b}) \cos q x\right. \\
& \left.\left.+\bar{P}_{1}(a, \bar{a}, b, \bar{b}) \cos q \xi\right)\right]
\end{aligned}
$$


where $P_{0}$ and $P_{1}$ are homogeneous polynomials of $a, b, \bar{a}$ and $\bar{b}$ of degree $2 q$ and $P_{0}$ is real valued. This is hardly surprising if we come back to the Floquet theory for the initial problem. The basic problem is of course to determine these two polynomials. We have already computed the case when $p=q=1$ and we found:

$$
P_{0}(a, \bar{a}, b, \bar{b})=2 \sin (2 \arg b), \quad P_{1}(a, \bar{a}, b, \bar{b})=|a||b| .
$$

It will be quite interesting to have a general formula for these polynomials. Let us now compute in detail the case when $p=1 ; q=2$ which is the main object of this section. A tedious computation gives:

$$
\operatorname{det} Q_{0}(x, \xi)=2|b|^{2}|a|^{2}[\cos 2 x+\cos 2 \xi]+2 \cos (4 \arg b)+2\left(|a|^{2}-|b|^{2}\right)
$$

or

$$
\begin{aligned}
\operatorname{det} Q_{0}(x, \xi) / 2= & \frac{1}{4}\left(1-\tanh ^{2}\left(\frac{\pi \mu^{\prime}}{h}\right)\right)[\cos 2 x+\cos 2 \xi] \\
& +\cos (4 \arg b)-\tanh \left(\frac{\pi \mu^{\prime}}{h}\right) .
\end{aligned}
$$

Another way to write the second term of the r.h.s is:

$$
2 \mathfrak{R}\left(a^{4}+b^{4}-2 a^{2} b^{2}\right)+2|a|^{4}-2|b|^{4} .
$$

We observe now that we are essentially in the same situation as in the preceding section. Modulo the control of the remainder term, we find:

$$
\mu^{\prime} \in \tilde{\sigma}_{h} \text { iff } \cos (4 \arg b) \in\left[-\frac{1}{2}, \frac{1}{2}\right]\left(1-\tanh ^{2} \frac{\pi \mu^{\prime}}{h}\right)+\tanh \frac{\pi \mu^{\prime}}{h}
$$

As in the preceding section we obtain:

$$
\begin{array}{r}
\frac{\left|\sigma_{h}\right|}{h} \rightarrow \int_{-\infty}^{+\infty}\left[\arccos \left(-\frac{1}{2}+\frac{1}{2} \tanh ^{2} \pi s+\tanh \pi s\right)\right. \\
\left.\quad-\arccos \left(\frac{1}{2}-\frac{1}{2} \tanh ^{2} \pi s+\tanh \pi s\right)\right] d s
\end{array}
$$

We set $t=\tanh \pi s$ and get:

$$
\frac{\left|\sigma_{h}\right|}{h} \rightarrow \frac{I}{\pi^{2}}
$$

with

$$
I=\int_{-1}^{+1}\left[\arccos \left(-\frac{\left(1-t^{2}\right)}{2}+t\right)-\arccos \left(\frac{\left(1-t^{2}\right)}{2}+t\right)\right] \frac{d t}{1-t^{2}}
$$


We now use the following formula:

$$
\begin{aligned}
& {\left[\arccos \left(-\frac{\left(1-t^{2}\right)}{2}+t\right)-\arccos \left(\frac{\left(1-t^{2}\right)}{2}+t\right)\right]} \\
& =\int_{-1}^{+1}-\partial / \partial u \arccos \left(u \frac{\left(1-t^{2}\right)}{2}+t\right) d u \\
& =\int_{-1}^{+1} \frac{1-t^{2}}{2\left[1-\left(u \frac{\left(1-t^{2}\right)}{2}+t\right)^{2}\right]^{\frac{1}{2}}} d u .
\end{aligned}
$$

With $t^{\prime}=t-\frac{1}{u}$ we can rewrite the term between the brackets as:

$$
\left[1-\left(u \frac{\left(1-t^{2}\right)}{2}+t\right)^{2}\right]=-\frac{u^{2}}{4}\left(\left(1-\frac{1}{u}\right)^{2}-t^{\prime 2}\right)\left(\left(1+\frac{1}{u}\right)^{2}-t^{\prime 2}\right) \text {. }
$$

With $a(u)=-1-\frac{1}{u}, b(u)=1-\frac{1}{u}$, we then obtain:

$$
I=\int_{-1}^{+1} d u \int_{a(u)}^{b(u)} \frac{d t^{\prime}}{|u|\left[\left(t^{\prime 2}-a^{2}(u)\right)\left(b^{2}(u)-t^{\prime 2}\right)\right]^{\frac{1}{2}}} .
$$

We now use the following change of variables $t^{\prime 2}=\frac{a^{2}+b^{2}}{2}+\frac{b^{2}-a^{2}}{2} \cos \theta$ with $\theta \in[0, \pi]$ and get:

$$
\begin{aligned}
I & =\int_{-1}^{+1} \int_{0}^{\pi} \frac{d \theta d u}{2|u|\left(\frac{a(u)^{2}+b(u)^{2}}{2}+\frac{b(u)^{2}-a(u)^{2}}{2} \cos \theta\right)^{\frac{1}{2}}} \\
& =\int_{-1}^{+1} \int_{0}^{\pi} \frac{d \theta d u}{2|u|\left(1+\frac{1}{u^{2}}-\frac{2}{u} \cos \theta\right)^{\frac{1}{2}}}=\int_{-1}^{+1} \int_{0}^{\pi} \frac{d \theta d u}{2 \sin \theta\left[\left(\frac{u}{\sin \theta}-\frac{\cos \theta}{\sin \theta}\right)^{2}+1\right]^{\frac{1}{2}}} \\
& =\int_{0}^{\pi}\left[\sinh ^{-1}\left(\frac{u}{\sin \theta}-\frac{\cos \theta}{\sin \theta}\right)\right]_{u=-1}^{u=1} \frac{d \theta}{2}=\int_{0}^{\pi}\left[\sinh ^{-1} \tan \frac{\theta}{2}+\sinh ^{-1} \cot \frac{\theta}{2}\right] \frac{d \theta}{2} \\
& =\int_{0}^{\pi}\left[\sinh ^{-1} \cot \frac{\theta}{2}\right] d \theta .
\end{aligned}
$$

But

and we get

$$
\sinh ^{-1} \cot \frac{\theta}{2}=\ln \cot \frac{\theta}{4}
$$

$$
I=\int_{0}^{\pi} \ln \cot \frac{\theta}{4}
$$

A new change of variables $s=\tan \frac{0}{4}$ gives finally:

$$
I=\int_{0}^{1}-\ln s \frac{4 d s}{1+s^{2}}=4 \int_{0}^{1} \frac{\arctan s}{s} d s .
$$


Hence (comparing with (3.20)-(3.21))

$$
\frac{\left|\sigma_{h}\right|}{h} \rightarrow \frac{4 C_{C a t}}{\pi^{2}}
$$

for $h=2 \pi \cdot \frac{2}{q}$ and consequently:

$$
q\left|\sigma_{\frac{4 \pi}{q}}\right| \rightarrow \frac{16 C_{C a t}}{\pi}
$$

as $q \rightarrow \infty$ with $q \wedge 2=1$. We have finally proved the theorem (modulo the control of the effect of the exponentially small remainder term). But this last problem can be treated as in the preceding section using the selfadjointization procedure.

\section{Extensions, Open Problems and Conclusion}

Our proof suggests generalizations.

(1) A weaker result than Thouless conjecture will be to prove simply the existence of the limit without computing it. This seems possible to obtain for the sequence $\alpha=\frac{1}{q+\frac{1}{r}}$. For $r=3$, we get the following condition (forgetting exponentially small errors) which seems more difficult to analyze than in the cases $r=2$ and $r=\infty$ :

$\mu \in \operatorname{Sp} H$ iff $\sin (6 \gamma)-3|a|^{4} \sin (2 \gamma+2 \pi / 3)-3|b|^{4} \sin (2 \gamma-2 \pi / 3) \in[-2,2]|a b|^{3}$,

with $\gamma=\arg a+\pi / 3$ and $\mu^{\prime}=f(\mu, h)$. This formula looks relatively simple but it seems difficult to do the analog of the computations performed in the preceding sections.

(2) Following [17] and [18], it is also possible to approach in the same spirit the case of the hexagonal Harper's equation.

(3) Another point is that Y. Last and M. Wilkinson give in [21] heuristic arguments in order to deduce from the Thouless conjecture a new conjecture concerning any rational with large denominator. A little more precisely, the statement is the following vague

Conjecture 5.1. If $p_{n} / q_{n}$ is irreducible and tends (sufficiently rapidly?) to a rational $p_{0} / q_{0}$ with $q_{n} \rightarrow \infty$, then

$$
\lim _{n \rightarrow \infty} q_{n}\left|\Sigma\left(p_{n} / q_{n}, 1\right)\right|=16 C_{C a t} / \pi .
$$

We note here that all these conjectures have been tested numerically in $[25,26$ or 21]. Let us for example try to prove the following "theorem" due to [21]:

Theorem 5.2. Let us assume that

$$
\frac{h}{2 \pi}=\left[q_{1}, q_{2}, \ldots, q_{n}, s\right]=\frac{p(s)}{q(s)},
$$

then

$$
\lim _{s \rightarrow \infty} q(s)|\Sigma(h, 1)|=(16 / \pi) C_{\text {Cat }} .
$$

The proof of this "theorem" which is given in [21] is only heuristic. We shall now explain how this strategy can be made more rigorous (but a complete rigorous proof 
will be probably quite long and has not been completely achieved and this is why we write "Theorem" instead of Theorem) using [14] and [13]. The starting point is to proceed using the semi-classical analysis near a rational $\alpha_{\infty}$ which is here:

$$
\alpha_{\infty}=\left[q_{1}, q_{2}, \ldots, q_{n}\right]=\frac{p(\infty)}{q(\infty)} .
$$

We recall also that Bezout's Theorem gives the existence of two integers $l(p)$ and $n(p)$ with $l(p) \in]-q, 0]$ such that:

$$
p \cdot l(p)+q \cdot n(p)=-1 .
$$

With this choice we have also

$$
p(s)=s p-n(p), \quad q(s)=s q+l(p) .
$$

Let us also introduce

$$
\delta=\frac{h}{2 \pi}-\alpha_{\infty} .
$$

In this case it is proved by Helffer-Sjöstrand in [13], justifying previous mathematically non-rigorous works of Wilkinson [32,33] and Sokoloff [24], that the study of the spectrum is reduced to the study of the spectrum of a $\delta$-pseudodifferential system whose symbol is given by $\frac{1}{2} M_{p, q}(x, \xi)$, where

$$
M_{p, q}(x, \xi)=\left(K \exp i x+K^{-1} \exp -i x+J^{p} \exp i \xi+J^{-p} \exp -i \xi\right),
$$

with $p=p(\infty), q=q(\infty)$ and where $J$ and $K$ are introduced in (4.3). Let us also recall that:

$$
\operatorname{det}\left(M_{p, q}(x, \xi)-E\right)=f_{p, q}(E)+2(-1)^{q}(\cos q x+\cos q \xi) .
$$

The following identity of [21] will be crucial in the proof:

$$
\sum_{v=1}^{q} \frac{1}{\left|f_{p, q}^{\prime}\left(E_{v}\right)\right|}=\frac{1}{q}
$$

where $E_{v}$ are the distinct zero's of the polynomial $f_{p, q}$.

We use now the rigorous reduction of the problem near each band. Everything is concentrated at the middle of each band, that is near the points $E_{v}$. Let $t \rightarrow E_{v}(t)$ the branch of solution of the equation:

$$
f_{p, q}\left(E_{v}(t)\right)=(-1)^{q+1} t, \quad E_{v}(0)=E_{v} .
$$

Then the decoupling procedure introduced in [13] says that we have to study a $\delta$-pseudodifferential operator whose principal symbol is:

$$
(x, \xi) \rightarrow \frac{1}{2} E_{v}(2(\cos q x+\cos q \xi))
$$

with

$$
q=q(\infty) .
$$

The study of the operator near the energy corresponding to the saddle point can probably be reduced to microlocal considerations as in [14] but this point is not 
discussed in [13]. We just sketch here the discussion which explains our "probably." In order to study microlocally the $\delta$-pseudodifferential operator we perform a symplectic dilation and arrive at a $\hat{h}$-pseudodifferential operator with $\hat{h}=q^{2} \delta$ and with the new principal symbol $\frac{1}{2} E_{v}(2 \cos x+2 \cos \xi)$. For $\hat{h}$ small enough we can apply the discussion of [13]. The function $t \rightarrow f(t, \hat{h})$ introduced in order to get a fixed period equal to $2 \pi$ is no more satisfying $(2.5) f_{0}(0)=0 ; f_{0}^{\prime}(0)=1$ but

$$
f_{0}(0)=E_{v}, \quad f_{0}^{\prime}(0)=\left|f_{p, q}^{\prime}\left(E_{v}\right)\right|
$$

and this just changes the measure asymptotically by a fixed factor $\left|f_{p, q}^{\prime}\left(E_{v}\right)\right|^{-1}$. Let us now give the formula for $\hat{h}$ :

$$
\hat{h}(s)=\frac{q^{2} \delta}{2 \pi}=q^{2}(\alpha(s)-\alpha(\infty))=\frac{q}{s q+l} .
$$

Here we have used the part of the proof which is mentioned in Remark 2.4. The second point corresponds to Remark 2.6. $h^{\prime}$ has to be replaced by $\tilde{h}$ which is found by determination of the two operators $\mathscr{T}$ and $\mathscr{T}^{\star}$ playing in this new context the role of $\tau$ and $\tau^{\star}$. These operators $\mathscr{T}$ and $\mathscr{T}^{\star}$ are introduced in the same context in [13] exploiting of course previous ideas of physicists (see for example [23, 24, 32, 33 and 3]) and defined by:

$$
\begin{aligned}
\mathscr{T} & =\tau_{2 \pi / q} J, \\
\mathscr{T}^{\star} & =\tau_{2 \pi / q}^{\star} K^{l(p)},
\end{aligned}
$$

where

$$
\left(\tau_{\frac{2 \pi}{q}} u\right)(x)=u(x-2 \pi / q), \quad\left(\tau_{\frac{2 \pi}{q}}^{\star}\right) u(x)=\left(\exp \frac{2 i \pi x}{q}\right) u(x) .
$$

We observe, using (4.3) and (4.6), that these operators commute with $M_{p, q}\left(x, \delta D_{x}\right)$ and satisfy the relation (to compare with 2.20 )

$$
\mathscr{T} \mathscr{T}^{\star}=\exp -i \tilde{h} \mathscr{T}^{\star} \mathscr{T}
$$

with

$$
\frac{\tilde{h}}{2 \pi}=\frac{2 \pi}{q^{2} \delta}-\frac{l(p)}{q}(\bmod (2 \pi \mathbb{Z})) .
$$

We can then reduce (modulo some technical details that we have not completely verified) the study of $M\left(x, \delta D_{x}\right)$ (here we come back to a global renormalization procedure) near the energy $E_{v}$ to the study of the $h$-pseudodifferential operator $Q$ whose symbol was introduced in Theorem 2.1 with a different $g$ and with a new $f$ defined above. But for our particular choice of $h$ we get $\tilde{h}=0$ and we are consequently in the case of Sect. 2.

We note here that the second term $-\frac{l(p)}{q}$ in the definition of $h$ is specific of the case of systems. This is also related to the non-triviality of the fiber bundle above the torus whose fiber is

$$
\operatorname{Ker}\left(M_{p, q}(x, \xi)-E_{v}(2 \cos q x+2 \cdot \cos q \xi)\right),
$$


(see for example [15] for a discussion in the same spirit or [5] (and the bibliography) for other references in connection with the study of the quantum Hall effect). The statement we need to give a complete mathematical proof is unfortunately not established in [13] (Proposition 5.4.1) which gives only the equivalent of Theorem 1.5 but does not analyze the contribution of the middle. We consequently have to implement the results of [14] inside the machinery of [13]. According to our three remarks in Sect. 2 and to what we said above, this seems reasonable but it would be probably rather long to write down all the mathematical details (mainly the remainder estimates). If $S_{v}(s)$ is the total bandwidth for this effective hamiltonian we finally get

$$
\lim _{s \rightarrow \infty} \hat{h}(s) S_{v}(s)=\frac{1}{\left|f_{p, q}^{\prime}\left(E_{v}\right)\right|} \frac{16 C_{c a l}}{\pi} .
$$

Here we have observed that

$$
\lim _{s \rightarrow \infty} h(s) / s=1 .
$$

Using now (5.5) which implies

$$
\lim _{s \rightarrow \infty} q(s) / s=q(\infty)=q,
$$

we get:

$$
\lim _{s \rightarrow \infty} q(s) S_{v}(s)=\frac{q}{\left|f_{p, q}^{\prime}\left(E_{v}\right)\right|} \frac{16 C_{c a t}}{\pi} .
$$

Summing the different contributions of each band and using (5.9), we get the "Theorem" 5.2. Hence the strategy described by Y. Last and M. Wilkinson seems to us to lead to a correct mathematical proof. As observed by these authors this gives a direct connection between the proof of the conjecture near 0 and the proof of the conjecture near a rational number.

Acknowledgements. We want to thank Y. Last for motivating discussions with the first author at the conference in Vancouver (August 1993). We thank also J. Sjöstrand for previous collaborations on this subject where most of the ideas presented here had been already developed. Finally we thank S. Fedotov for interesting discussions and comments on this article.

Note added in proof. We recently discover that due to a material error in the edition the paging in $[15]$ is wrong. The good order for reading this paper is ...28-29-37-36-35-34-33-32-31-30-38-39...

\section{References}

1. Avron, J., van Mouche, P., Simon, B.: On the measure of the spectrum for the almost Mathieu operator. Commun. Math. Phys. 132, 103-118 (1990)

2. Azbel, Ya.: Energy spectrum of a conduction electron in a magnetic field. Sov. Phy. JETP19, $n^{0} 3$ (1964)

3. Bellissard, J.: $\mathrm{C}^{\star}$ algebras in solid state physics (2D electrons in a uniform magnetic fields). Warwick Conference on Operators Algebras (1987)

4. Bellissard, J.: Almost periodicity in solid state physics and $\mathrm{C}^{\star}$-algebras. In: Berg, C., Flugede, B. (eds.) The Harald Bohr centenary. The Danish Royal Acad. Sci. 42.3, 35-75 (1989)

5. Bellissard, J.: Le papillon de Hofstadter. Séminaire Bourbaki, $44^{e ̀ m e}$ année, $n^{0} 745$ (1991)

6. Bellissard, J., Simon, B.: Cantor spectrum for the almost Mathieu equation. J. Funct. An. 48, $n^{0} 3$ (1982)

7. Buslaev, V., Fedotov, S.: Complex WKB method for the Harper's equation. Preprint of the Mittag-Leffler Institute, $n^{0} 11$ (1993) 
8. Buslaev, V., Fedotov, S.: The functional structure of the monodromy matrix for Harper's equation. To appear in Operator Theory: Advances and applications (1994)

9. Buslaev, V., Fedotov, S.: On a class of matrices related to Harper's equation. Preprint of the Mittag-Leffler Institute, $n^{0} 18$ (1993)

10. Choi, M.D., Elliott, G.A., Yui, N.: Gauss polynomials and the rotation algebra. Invent. Math. 99, 225-246 (1990)

11. Guillement, J.P., Helffer, B., Treton, P.: Walk inside Hofstadter's butterfly. J. Phys. France 50, 2019-2058 (1989)

12. Helffer, B., Sjöstrand, J.: Analyse semi-classique pour l'équation de Harper (avec application à l'étude de Schrödinger avec champ magnétique). Mémoire de la SMF $n^{0} 34$; Tome 116, Fäsc. 4 (1988)

13. Helffer, B., Sjöstrand, J.: Analyse semi-classique pour l'équation de Harper II (comportement semi-classique près d'un rationnel). Mémoire de la SMF $n^{0} 40$; Tome 118, Fasc. 1 (1990)

14. Helffer, B., Sjöstrand, J.: Semi-classical analysis for Harper's equation III. Mémoire de la SMF $n^{0} 39$, Tome 117, Fasc. 4 (1989)

15. Helffer, B., Kerdelhué, P., Sjöstrand, J.: Le papillon de Hofstadter revisité. Mémoire de la SMF $n^{0} 43$, Tome 118 , Fasc. 3 (1990)

16. Hofstadter, D.: Energy levels and wave functions for Bloch electrons in rational and irrational magnetic fields. Phys. Rev. B 14, 2239-2249 (1976)

17. Kerdelhué, P.: Spectre de l'opérateur de Schrödinger magnétique avec symétrie d'ordre 6. Mémoire de la SMF $n^{0} 51$; Tome 120, Fasc.4, (1992)

18. Kerdelhué, P.: Equation de Schrödinger magnétique avec symétrie d'ordre 6: Mesure du spectre II. Preprint Université Paris-Sud (1993) to appear in mémoires de l'IHP, Phys. Th.

19. Last, Y.: A relation between a.c. spectrum of ergodic Jacobi matrices and the spectra of periodic approximants. Commun. Math. Phys. 151, 183-192 (1993)

20. Last, Y.: Zero measure spectrum for the almost Mathieu operator. Commun. Math. Phys. 164, 421-432 (1994)

21. Last, Y., Wilkinson, M.: A sum rule for the dispersion relations of the rational Harper equation. J. Phys. A: Math. Gen. 25, 6123-6133 (1992)

22. Van Mouche, P.: The coexistence problem for the discrete Mathieu operator. Commun. Math. Phys. 122, 23-34 (1989)

23. Sokoloff, J.B.: Phys. Rev. B 23, 2039-2041 (1981)

24. Sokoloff, J.B.: Unusual band structure, wave functions and electrical conductance in crystals with incommensurate periodic potentials. Phys. Reports (review section of Phys. Lett.), 126, $n^{0} 4,189-244$ (1985)

25. Yong Tan, Thouless, D.J.: Total bandwidth for the Harper equation III. Corrections to scaling. J. Phys. A: Math. Gen. 24, 4055-4066 (1991)

26. Yong Tan, Thouless, D.J.: Total energy for two dimensional lattice fermions in a magnetic field. Physica A177, 567-577 (1991)

27. Thouless, D.J.: Bandwidths for a quasiperiodic tight binding model. Phys. Rev. 28, 4272-4276 (1983)

28. Thouless, D.J.: Commun. Math. Phys. 122, 23-34 (1989)

29. Thouless, D.J.: Scaling for the discrete Mathieu equation. Commun. Math. Phys. 127, 187-193 (1990)

30. Thompson, C.J.: Mathematical statistical mechanics. Princeton, NJ: Princeton University Press, 1972

31. Watson, G.I.: WKB analysis of energy band structure of modulated systems. J. Phys. A24, 4999-5010 (1991)

32. Wilkinson, M.: Critical properties of electron eigenstates in incommensurate systems. Proc. R. Soc. London A391, 305-350 (1984)

33. Wilkinson, M.: An example of phase holonomy in WKB theory. J. Phys. A. Math. Gen. 17, 3459-3476 (1984)

34. Wilkinson, M.: An exact renormalisation group for Bloch electrons in a magnetic field. J. Phys. A 20, $n^{0} 7,1791$ (1987) 